\title{
Predictive value of cytokine levels for acute graft-versus-host disease in he- matopoietic stem cell transplantation with post-transplant cyclophosphamide
}

\author{
Olga V. Pirogova ${ }^{1}$, Ivan S. Moiseev ${ }^{1}$, V. V. Beklenischev ${ }^{1}$, Elena A. Surkova ${ }^{2}$, Sergey V. Lapin ${ }^{2}$, Elena V. Babenko ${ }^{1}$ Alexan- \\ der L. Alyanskiy ${ }^{1}$, Boris V. Afanasyev ${ }^{1}$
}

${ }^{1}$ R.M. Gorbacheva Memorial Institute of Children Hematology, Oncology and Transplantation; ${ }^{2}$ Laboratory of Autoimmune Diagnostics, First St.Petersburg State I.P. Pavlov Medical University, St. Petersburg, Russian Federation

Contact: Dr. Olga V. Pirogova

E-mail: dr.pirogova@gmail.com

\section{Background}

Currently, acute graft-versus-host-disease (GVHD) remains one of the most serious complications of allogeneic hematopoietic stem cell transplantation (HSCT). The release of large amounts of cytokines causes activation of alloreactive T-cells in the early period after transplantation is an important step in pathogenesis of acute GVHD. The basic immunological events leading to GVHD occur during several weeks after hematopoietic graft transfusion. By this reason, cytokine profiling could be used to predict development of GVHD. The majority of studies on cytokines in allogeneic HSCT were performed with classical GVHD prophylaxis, consisting of non-specific immunosuppressive agents like calcineurin inhibitors, methotrexate, mycophenolate mofetil, anti-thymocyte globulin, etc. When using this type of prophylaxis, most studies agree in that higher levels of pro-inflammatory cytokines are associated with development of acute GVHD, while lower levels indicate a successful immunosuppression in abrogation of alloreactive response. Recently, many transplant centers are interested in a novel approach for the GVHD prevention based on Cyclophosphamide post-HSCT (PTCy). Currently, there is no clarity, whether cytokine changes after PTCy are similar to the situation with classical GVHD prophylaxis. It is also unclear if well-known predictive biomarkers remain valid after PTCy-based prophylaxis. Trying to answer these questions, we have conducted a pilot study to determine the dynamics of plasma cytokines in patients undergoing HSCT with PTCy for GVHD prophylaxis. For this pilot study, we have selected five cytokines, IL17, IL-6, IL-8, IFN- $\gamma$, and TNF- $\alpha$, that were shown to have predictive value for acute GVHD. We, therefore, compared their levels in patients who developed acute GVHD versus GVHD-free cases.

\section{Patients and Methods}

Among 192 patients transplanted with PTCy prophylaxis at The First State I.P. Pavlov Medical University between 2014 and 2015, we have identified 20 cases with acute GVHD and plasma samples available. These patients were matched to patients who did not develop acute GVHD at a 1:2 ratio. By decreasing priority, the matching criteria were as follows: donor type, graft source, intensity of the conditioning regimen, primary diagnosis, and disease status. Thus, the study group consisted of sixty adult patients with hematological malignancies who underwent HSCT. All patients have signed informed consent for the collection of blood samples and use of their personal data for research purposes. $28 \%$ were grafted from related donor, $72 \%$ - from unrelated. The patients received either myeloablative conditioning (MAC) with oral Busulfan (16 $\mathrm{mg} / \mathrm{kg}$ ) and Cyclophosphamide (100 mg/kg), or reduced-intensity conditioning (RIC) with oral Busulfan $8 \mathrm{mg} / \mathrm{kg}$ and Fludarabine $180 \mathrm{mg} / \mathrm{m}^{2}$. All the patients received PTCy- based GVHD prophylaxis. For recipients transplanted from 10/10HLA matched unrelated donors (MUD), GVHD prophylaxis included combination of Cyclophosphamide $(50 \mathrm{mg} / \mathrm{kg})$ on day $+3,+4$, Tacrolimus with target concentration of 5-15 ng/ $\mathrm{ml}$ from day +5 through day+120, and Mycophenolate Mofetil (MMF) at $30 \mathrm{mg} / \mathrm{kg}$, or $45 \mathrm{mg} / \mathrm{kg}$ for 8-9/10 mismatched unrelated donors (MMUD) from day+5 through day+35. For matched related donors (MRD) with bone marrow as graft source, GVHD prophylaxis was a single-agent Cyclophosphamide $(50 \mathrm{mg} / \mathrm{kg})$ on day $+3,+4$. For blood testing, 10 $\mathrm{mL}$ of EDTA-preserved venous blood were obtained from the patients on days $-7,0,+7,+21$ and +28 . Plasma samples were obtained by centrifugation at $1000 \mathrm{~g}$, and then stored in aliquotes at $-80^{\circ} \mathrm{C}$ until the day of assays. We studied 5 plasma biomarkers, i.e., IL-17A, IL-6, IL-8, TNF- $\alpha$ and IFN- $\gamma$. 
Appropriate plasma levels were measured with ELISA technique using commercially available kits (Cytokine, St.Petersburg, Russia). All the assays were performed in compliance with protocols provided by the kit manufacturer. The cytokine concentrations were measured without knowledge of individual clinical data.

\section{Results}

Of sixty patients included into the study, twenty recipients developed aGVHD grade I-IV, whereas forty patients were GVHD-free. Eight of these 20 patients (40\%) had grade I; 7 (35\%) grade II; 5 (25\%) grade III aGVHD. 6 patients $(30 \%)$ developed a systemic aGVHD. There was no difference in cytokine concentrations between GVHD+ and GVHDgroups. The levels of all the cytokines under study did not show any correlations with a risk for aGVHD grades I-IV. We have not received significant differences between the concentrations of cytokines in the study groups at any time point $(p>0.05)$. Noteworthy, additional comparisons between the groups with systemic GVHD and without GVHD have shown lower levels of several plasma cytokines in patients who did develop GVHD. A reduced mean level of IL-8 (98.74 \pm 39.47 vs $276.09 \pm 284.78 \mathrm{pg} / \mathrm{ml}, \mathrm{p}=0.008$ for GVHD+ and GVHD-, respectively), and IFN- $\gamma(22.69 \pm 16.35$ vs $60.07 \pm 42.00 \mathrm{pg} / \mathrm{ml}, \mathrm{p}=0.005$ for GVHD+ and GVHD-, re- spectively) at day +28 were significant risk factors for aGVHD occurrence. By ROC analysis, cutoff values were determined for IL-8, as (147.09 pg/ml; AUC=0.833), and for IFN- $\gamma(35.94 \mathrm{pg} / \mathrm{ml} ; \mathrm{AUC}=0.848)$. Incidence of systemic aGVHD was significantly higher in patients with cytokine levels under the cutoff values ( $0 \%$ vs $35.3 \%, \mathrm{p}=0.002$, and $3.8 \%$ vs $38.5 \%, p=0,003$, for IL- 8 and IFN- $\gamma$, respectively).

\section{Conclusions}

In summary, our pilot trial has shown that time dynamics of cytokine levels after GVHD prophylaxis with PTCy might be different from those under conventional immunosuppressive treatment. Likewise, the well known predictive biomarkers might not work after PTCy. Further large prospective trials could elucidate the differences and determine reliable predictors for acute GVHD. Moreover, it also may be important to introduce additional time points for blood sampling before PTCy administration.

\section{Keywords}

Allogeneic hematopoietic stem cell transplantation, GvHD prevention, posttransplant Cyclophosphamide, cytokines, blood plasma.

\section{Прогностическая значимость уровней цитокинов для острой реакции «трансплантат против хозяина» при трансплантации гемопоэтических стволовых клеток с посттрансплантационным применением циклофосфамида}

Ольга В. Пирогова ${ }^{1}$, Иван С. Моисеев ${ }^{1}$, В.В. Бекленищев ${ }^{1}$, Елена А. Суркова ${ }^{2}$ Сергей В. Лапин ${ }^{2}$, Елена В. Бабенко ${ }^{1}$, Александр Л. Алянский ${ }^{1}$ Борис В. Афанасьев ${ }^{1}$

${ }^{1}$ НИИдетской онкологии, гематологии и трансплантологии им. Р.М. Горбачевой.; ${ }^{2}$ Лаборатория диагностики аутоиммунных заболеваний, Первый Санкт-Петербургский государственный медицинский университет им. акад. И. Павлова, СанктПетербург, Российская Федерация

\section{Введение}

Острая реакция «трансплантат против хозяина» (оРТПХ) acute graft-versus-host-disease остается одним из наиболее серьезных осложнений аллогенной трансплантации гемопоэтических стволовых клеток (ТГСК). Секреция больших количеств цитокинов вызывает активацию аллореактивных Т-клеток в ранние сроки после ТГСК является важным этапом в патогенезе оРТПХ. Основные иммунологические события, ведущие к
оРТПХ развиваются в течение нескольких недель после пересадки. По этой причине изучение профиля цитокинов могло бы применяться для прогнозирования РТПХ. Большинство исследований цитокинов при алло-ТГСК проводилось с классической профилактикой этого состояния, состоящей из неспецифических иммуносупрессивных агенто, в частности, ингибиторов кальцинейрина, метотрексата, микофенолята мофетила, антитимоцитарного глобулина и др. При использовании этого типа профилактики, многие исследования согласны в том, что повышенные уровни провоспалительных 
цитокинов связаны с развитием острой РТПХ, тогда как более низкие уровни указывают на эффективную иммуносупрессию, предотвращающую аллореактивный ответ. С недавних пор многие трансплантационные центры интересуются новым подходом профилактики РТПХ, основанным на введении циклофосфамида после ТГСК (ПТЦф). В настоящее время нет ясности относительно того, сходны ли здесь изменения цитокинового фона с тем, что наблюдается при классической профилактике РТПХ. Неясно также, остаются ли валидными известные прогностические маркеры после терапии ПТЦф. В попытке ответить на эти вопросы мы провели пилотное исследование цитокинов плазмы крови, чтобы определить их динамику у пациентов после ТГСК с применением ПТЦф для профилактики РТПХ. Для этого исследования мы выбрали пять цитокинов: IL-17, IL-6, IL-8, IFN- $\gamma$ и TNF- $\alpha$, которые, по ряду работ, имеют прогностическое значение при острой РТПХ. Поэтому мы сравнивали их уровни у пациентов, у которых развилась оРТПХ по сравнению со случаями без РТПХ.

\section{Пациенты и методы}

Среди 192 больных, трансплантированных с профилактикой РТПХ с помощью ПТЦф в Первом Санкт-Петербургском государственном медицинском университете им. И.П.Павлова в 2014-2015 гг., мы выявили 20 случаев оРТПХ, у которых исследовали образцы плазмы крови. Эти больные были сопоставимы с пациентами, у которых не развилась острая РТПХ, в соотношении 1:2. В порядке снижения приоритетности, критерии подбора были следующими: тип донора, источник трансплантата, интенсивность кондиционирующего режима, первичный диагноз и статус заболевания. Таким образом, общая группа состояла из 60 взрослых пациентов с онкогематологическими заболеваниями после ТГСК. Все пациенты подписывали информированное согласие на забор образцов крови и использование их персональных данных для научных целей. 28\% больных получили трансплантаты от родственного донора, 72\% от неродственных доноров. Больные получали либо миелоаблативный режим кондиционирования (МАК) с пероральным приемом бусульфана (16 мг/кг) и циклофосфамидом (100 мг/кг), либо кондиционирование сниженной интенсивности (RIC) с применением бусульфана перорально (8 мг/кг) и флударабина (180 мг/м²). Все пациенты ппроходили профилактику оРТПХ с применением ПТЦф. Для реципиентов, трансплантированных от HLA-совместимых по 10/10 антигенов неродственных доноров (MUD), профилактика РТПХ включала сочетание циклофосфамида $(50 \mathrm{Mг} /$ кг) в день $+3,+4$; такролимус с целевой концентрацией 5-15 нг/мл в сроки от дня +5 дня +120 и микофенолята мофетила (MMF) в дозе $30 \mathrm{мг/кг,} \mathrm{или} 45$ мг/кг для доноров, совместимых по 8-9/10 антигенам (MMUD) со дня +5 до дня+35. В случаях трансплантации костного мозга от родственных совместимых доноров (MRD) профилактика РТПХ была с применением одного агента - циклофосфамида (50 мг/кг) в день +3 и +4. Для исследования крови, 10 мл ЭДТА-стабилизированной венозной крови получали от больных в сроки $-7,0,+7,+21$ и +28 сут. Образцы плазмы получали центрифугировани- ем при $1000 \mathrm{~g}$, а затем хранили аликвоты при $-80^{\circ} \mathrm{C}$ до анализа. Мы изучали в плазме 5 биомаркеров, а именно IL-17A, IL-6, IL-8, TNF- $\alpha$ и IFN- $\gamma$. Уровни цитокинов в плазме определяли с помощью ИФА, с применением коммерческих наборов («Цитокин», Санкт-Петербург, Россия). Все анализы проводили в соответствии с протоколами от производителя. Концентрации цитокинов измеряли без информации о клинических данных пациентов.

\section{Результаты}

Из 60 больных, включенных в исследование, у 20 реципиентов развилась оРТПХ различной степени (I-IV), тогда как у 40 больных РТПХ не отмечалась. Восемь из этих 20 паиентов (40\%) имели РТПХ І степени; 7 (35\%) II степени; 5 (25\%) - III степени. У 6 пациентов (30\%) развилась системная оРТПХ. Нами не выявлено различий в концентрациях цитокинов между группами с РТПХ и без нее. Уровни всех исследованных цитокинов не коррелировали с риском развития РТПX I-IV степени. Мы не выявили достоверных различий по цитокинам в этих группах в любые сроки наблюдения (p>0.05). Интересно, что дополнительные сравнения между группами с системной РТПХ и без РТПХ показали снижение уровней некоторых цитокинов в плазме у пациентов с РТПХ. Так, снижение среднего уровня у больных с РТПХ показано для IL-8 (соответственно, 98,74 $\pm 39,47$ против $276,09 \pm 284,78$ пг/мл, p=0,008), и IFN- $\gamma(22,69 \pm 16,35$ против $60,07 \pm 42,00$ пг/мл, $\mathrm{p}=0.005)$ в день +28 , что явилось существенным фактором риска развития острой РТПХ. C помощью ROC-анализа были определены граничные пороги (cut-off values) для IL-8 (147,09 пг/мл; AUC= $0,833)$ и для IFN- $\gamma(35,94$ пг/мл; $\mathrm{AUC}=0.848)$. Частота возникновения системной оРТПХ была значительно выше у пациентов с уровнями цитокинов ниже граничных порогов (0\% против $35,3 \%, \mathrm{p}=0.002$, и $3,8 \%$ против $38,5 \%, \mathrm{p}=0,003$, соответственно, IL-8 и IFN- $\gamma$ ).

\section{Выводы}

В целом, наше пилотное исследование показало, что временная динамика уровней цитокинов после профилактики оРТПХ с применением циклофосфамида (ПТЦф) может отличаться от таковой при обычной иммуносупрессивной терапии. Также показано, что хорошо известные прогностические маркеры могут не работать после ПТЦф. Дальнейшие проспективные исследования могут прояснить эти различия и определить надежные прогностические признаки острой РТПХ. Кроме того, может быть важным введение дополнительных временных точек для взятия крови перед назначением профилактики ПТЦф.

\section{Ключевые слова}

Аллогенная трансплантация гемопоэтических клеток, профилактика РТПХ, циклофосфамид, цитокины, плазма крови. 